\title{
PRE-CONFERENCE WORKSHOP
}

\section{XML/TEI Workshop}

\section{Learn How to Encode Documents and \\ Contribute to the Wesley Works Digital Edition}

Andrew Keck, Southern Methodist University

Michelle M. Taylor, University of South Florida

ABSTRACT: In this session, in many ways a follow-up to last year's Atla session "Proposing a TEI-Encoding Project for the Wesley Works," we introduced participants to the principles of text encoding with XML/TEI. While last year we discussed the rationale for using TEI to create a digital version of the Bicentennial Edition of the Works of John Wesley, as well as our plans for orchestrating such a large-scale project, this year we will offer introductory, hands-on training in TEI. Workshop participants will begin with the basics of text encoding common to any TEI project, then move on to a description of how the Wesley Works Digital Edition, specifically, has adopted and adapted these principles to meet its goal of creating a digital version of the Bicentennial Edition of the Works of John Wesley.

Our workshop was divided into three parts:

1) An introduction to the basic rules of eXtensible Markup Language (XML);

2) An introduction to the Text-Encoding Initiative (TEI), the species of XML developed for encoding literary and historical texts; and 
3) An overview of how documents (specifically, letters) are encoded in XML/TEI for inclusion in the Wesley Works Digital Edition.

While the first and second portions of the workshop were routine $\mathrm{XML} / \mathrm{TEI}$ training, the third and final portion was unique to Atla this year. This part was the result of almost a year's worth of developments in assessing encoding needs for Wesley's letters and beginning the encoding process, as we began with that genre and will be working on the volumes containing his correspondence for the next two to three years. While time limitations prevented our ability to go into as great an amount of detail as we preferred, we were able to go over both the main things one tags in a Wesley letter and the step-by-step process for tagging one. We outline these below.

\section{WHAT WE TAG WHEN WE TAG A WESLEY LETTER}

People (<persName> tag):

- The correspondent

- Anyone else referred to by name in the letter

- Anyone referred to in editorial notes

Places (<placeName> tag):

- The location from which the letter was posted

- Any other town/city or country mentioned in the letter

- Universities and colleges mentioned in the letter (e.g., Trinity College, Cambridge)

- Any other important named landmarks, whether residential or institutional (houses/estates, etc.)

Texts (various tags used):

Bible verses and bibliographic references to works both inside and outside the Wesley cannon.

Themes (<seg > tag):

Recurring themes; a more interpretive way to tag. 


\section{HOW TO CODE A WESLEY LETTER STEP BY STEP}

1) Open $X M L$ file containing letter template.

2) Open PDF containing the letter you want to encode (I have isolated a single letter).

3) Fill in TEI Header.

4) Fill in body of the letter by copying and pasting letter text, paragraph by paragraph, into your template.

5) You may choose to encode footnotes, page breaks, etc. at this point or at the end, whichever you prefer.

6) Add tags to all people and places (think proper nouns), using Excel spreadsheet to identify @xml:id for each person. NOTE: You can only use an @xml:id once per document, though you should use a <persName $>$ element at each occurrence of the name.

At the conclusion of our workshop, we gave participants a link to a survey on Google Forms where they could indicate interest in participating in the Wesley Works beginning in Fall 2021. 